\title{
Two-dimensional distributions and column densities of gaseous molecules in protoplanetary disks
}

\section{Deuterated species and UV shielding by ambient clouds}

\author{
Y. Aikawa ${ }^{1}$ and E. Herbst ${ }^{2}$ \\ 1 Department of Earth and Planetary Sciences, Kobe University, Kobe 657-8501, Japan \\ 2 Departments of Physics and Astronomy, The Ohio State University, Columbus, OH 43210, USA \\ e-mail: herbst@mps .ohio-state.edu
}

Received 22 November 2000 / Accepted 19 March 2001

\begin{abstract}
We have investigated the two-dimensional $(R, Z)$ distribution of deuterated molecular species in circumstellar disks around young stellar objects. The abundance ratios between singly deuterated and normal molecules ("D/H ratios") in disks evolve in a similar way as in molecular clouds. Fractionation is caused by rapid exchange reactions that are exothermic because of energy differences between deuterated and normal species. In the midplane region, where molecules are heavily depleted onto grain surfaces, the $\mathrm{D} / \mathrm{H}$ ratios of gaseous molecules are higher than at larger heights. The $\mathrm{D} / \mathrm{H}$ ratios for the vertical column densities of $\mathrm{NH}_{3}, \mathrm{H}_{2} \mathrm{O}$, and $\mathrm{HCO}^{+}$are sensitive to the temperature, and decrease significantly with decreasing radial distance for $R \lesssim 300$ AU. The analogous $\mathrm{D} / \mathrm{H}$ ratios for $\mathrm{CH}_{4}$ and $\mathrm{H}_{2} \mathrm{CO}$, on the other hand, are not very sensitive to the temperature in the range $(T=10-50 \mathrm{~K})$ we are concerned with, and do not decrease with decreasing $R$ at $R \geq 50 \mathrm{AU}$. The $\mathrm{D} / \mathrm{H}$ column-density ratios also depend on disk mass. In a disk with a larger mass, the ratios of deuterated species to normal species are higher, because of heavier depletion of molecules onto grains. In the second part of the paper, we report molecular column densities for disks embedded in ambient cloud gas. Our results suggest that CN and $\mathrm{HCO}^{+}$can be tracers of gaseous disks, especially if the central object is a strong X-ray source. Our results also suggest that the radial distributions of $\mathrm{CN}, \mathrm{C}_{2} \mathrm{H}, \mathrm{HCN}$, and $\mathrm{H}_{2} \mathrm{CO}$ may vary among disks depending on the X-ray luminosity of the central star.
\end{abstract}

Key words. ISM: molecules - stars: T Tauri - circumstellar matter - protoplanetary disk

\section{Introduction}

Protoplanetary disks are formed around young stellar objects as the stars are created, and are birthplaces of planetary systems. Molecular evolution in protoplanetary disks is important because it can reveal the chemical connection between planetary and interstellar matter. Since chemical abundances are determined by physical parameters such as temperature and density, studies of molecular evolution in protoplanetary disks can also be of help in determining the structure and physical evolution of the disks from the molecular line observations.

In recent years it has become possible to detect molecular lines in protoplanetary disks by radio astronomy. Aperture-synthesis images have directly revealed gaseous CO disks, which are in Keplerian rotation (Kawabe et al. 1993; Koerner et al. 1993; Dutrey et al. 1994; Koerner

Send offprint requests to: Y. Aikawa,

e-mail: aikawa@jet.planet.sci.kobe-u.ac.jp
\& Sargent 1995; Saito et al. 1995; Guilloteau \& Dutrey 1998). In addition to the CO studies, Dutrey et al. (1997) surveyed other molecular lines in the disks around DM Tau and GG Tau using the IRAM $30 \mathrm{~m}$ telescope. These stars, with ages $110^{6} \mathrm{yr}$ and $310^{5} \mathrm{yr}$ respectively (Beckwith et al. 1990; Handa et al. 1995), have large gaseous disks with radii $\sim 800 \mathrm{AU}$. The molecular line spectra detected towards the two stars are similar despite the fact that the disk around GG tau is circumbinary. The averaged fractional abundances of gaseous $\mathrm{CO}, \mathrm{CN}$, $\mathrm{CS}, \mathrm{HCN}, \mathrm{HNC}, \mathrm{H}_{2} \mathrm{CO}, \mathrm{C}_{2} \mathrm{H}$, and $\mathrm{HCO}^{+}$were reported by Dutrey et al. (1997), who found that the abundances of heavy-element-containing molecules relative to hydrogen are lower than those in molecular clouds by factors of 10-100, and relative abundances among heavy molecules are also different. Specifically, the abundance ratio of CN to $\mathrm{HCN}$ is much higher in disks than in molecular clouds.

In order to reveal the chemical and physical causes for these differences, Aikawa \& Herbst (1999a) (hereinafter 
Paper I) investigated the two-dimensional distribution of molecules in a disk by calculating molecular concentrations from a network of chemical reactions. They found molecular abundances to vary with height $Z$ from the midplane. At larger radii $(R \gtrsim 100 \mathrm{AU})$, which contribute most to the molecular emission lines because of the large area, the temperature is so low $(T \sim 10 \mathrm{~K})$, that most molecules, except for $\mathrm{H}_{2}$ and $\mathrm{He}$, are partially adsorbed onto grains to form ice mantles. In the midplane region $(Z \approx 0)$ molecular depletion is the most effective because disks are in hydrostatic equilibrium in the vertical direction, and the density is the highest in the midplane region.

In regions above and below the midplane, significant amounts of molecules remain in the gas phase because of lower densities, and because of non-thermal desorption, which is caused by cosmic rays and/or radiation (ex. X-rays) from the interstellar field and the central star. Aikawa \& Herbst (1999a) suggested that the observed molecular lines come mostly from this region. There is a height distinction between stable molecules and radicals, however. In the surface (uppermost) region of a disk, radicals such as $\mathrm{CN}$ are very abundant because of photodissociation via UV radiation. The molecular column densities obtained in Paper I by integrating over height are in reasonable agreement with observation; molecular depletion in the midplane explains the low average abundance of heavy-element-containing species relative to hydrogen, and the high abundance ratio of $\mathrm{CN}$ to $\mathrm{HCN}$ is caused by photochemistry in the surface region. It is clear that consideration of the 2-D distribution of molecules is essential in order to interpret the observed molecular line intensities.

In this paper we present two extensions of the work in Paper I. Firstly, we include deuterium chemistry in the 2-D disk model. Since the line survey by Dutrey et al. (1997), further searches for molecular lines in protoplanetary disks have been conducted by several groups of observers. One of the most interesting results is the detection of deuterated species in the disk of LkCa15 (Qi et al. 1999; Qi 2000). The average $\mathrm{DCN} / \mathrm{HCN}$ ratio is estimated to be about 0.01 , which is much higher than the $\mathrm{D} / \mathrm{H}$ elemental abundance ratio of $1.510^{-5}$. Aikawa \& Herbst $(1999 \mathrm{~b})$ showed that a high $\mathrm{D} / \mathrm{H}$ ratio in a disk is a natural outcome of the incorporation of interstellar material to the protoplanetary disk and a low temperature disk chemistry, which is similar to cloud chemistry. They were concerned, however, only with icy material in the midplane region, and did not predict column densities for deuterated gaseous molecules. A two-dimensional disk model with deuterium chemistry and comparison with observation should be helpful in constraining the physical properties of the disk, such as temperature. Such a theoretical model should also be useful in guiding searches for other deuterated molecules. The results presented here fulfill these expectations, at least partially.

Secondly, we consider disks that are embedded in molecular clouds or circumstellar envelopes. Up to now, molecular line observations of disks have been successful only in cases where a disk is removed from molecular clouds or where the system velocity is quite different from the cloud velocity. Most disks, which presumably are embedded in cloud gas or circumstellar envelopes, are hard to observe via molecular lines because of contamination with ambient gas. If we were able to predict which molecules could selectively trace disk gas, the species could be used to search for embedded gaseous disks, and so permit more statistically reliable arguments on disk properties, such as their size and time scales of gaseous dissipation.

The rest of the paper is organized as follows. In Sect. 2 we describe our model of protoplanetary disks and the chemical reaction network we utilize. Numerical results on the distribution of molecular abundances, the column density ratio of deuterated and normal species, and the dependence of $\mathrm{D} / \mathrm{H}$ ratios on selected physical properties of disks are discussed in Sect. 3. In Sect. 4, we report our investigation of molecular abundances in disks that are embedded in ambient gas, and discuss which, if any, molecular lines can be used as disk tracers. We summarize our results in Sect. 5.

\section{Model}

\subsection{Disk model}

As in Paper I, we have adopted simple static disk models - the Kyoto model (Hayashi 1981) extrapolated to $700 \mathrm{AU}$, and a model in which the mass and density are lowered by an order of magnitude. The lower mass model is similar to that adopted by Dutrey et al. (1997), with which they estimate the averaged molecular abundances in DM Tau. The total disk mass is about $610^{-2} M_{\odot}$ for the extended Kyoto model, and $610^{-3} M_{\odot}$ for the lower mass model. Although much work has been undertaken to formulate the structure and physical evolution of protoplanetary disks (Cameron 1973; Hayashi 1981; Adams \& Lin 1993, and references therein), there is still no obvious standard model. The main goal of this paper is to comprehend the essential characteristics of deuterium chemistry in a protoplanetary disk using simple disk models.

The details of the Kyoto model are described in Paper I. The column density of hydrogen nuclei $\Sigma_{\mathrm{H}}$ $\left(\mathrm{cm}^{-2}\right)$ and the temperature $T(\mathrm{~K})$ as functions of $R$ are given by the equations

$$
\begin{aligned}
\Sigma_{\mathrm{H}}(R) & =7.210^{23}\left(\frac{R}{100 \mathrm{AU}}\right)^{-3 / 2} \\
T(R) & =28\left(\frac{R}{100 \mathrm{AU}}\right)^{-1 / 2}\left(\frac{L_{*}}{L_{\odot}}\right)^{1 / 4} .
\end{aligned}
$$

The luminosity of the central star $L_{*}$ is assumed to be $1 L_{\odot}$ in this paper. It is assumed for simplicity that the disk is isothermal at each radius.

The gas is in hydrostatic equilibrium in the vertical direction. From the mass distribution given by Eq. (1) and the temperature distribution given by Eq. (2), the density 
distribution by number of hydrogen nuclei $\left(\mathrm{cm}^{-3}\right)$ can be shown to be

$$
\begin{aligned}
n_{\mathrm{H}}(R, Z)= & 1.910^{9}\left(\frac{R}{100 \mathrm{AU}}\right)^{-11 / 4} \exp \left(-\frac{G M_{*} \mu m_{\mathrm{H}}}{R k T}\right) \\
& \times \exp \left[\frac{G M_{*} \mu m_{\mathrm{H}}}{k T\left(R^{2}+Z^{2}\right)^{1 / 2}}\right]
\end{aligned}
$$

where $G$ is the gravitational constant, $\mu$ is the mean molecular weight of gas, $m_{\mathrm{H}}$ is the mass of a hydrogen atom, and $k$ is the Boltzmann constant. Since the disk mostly consists of $\mathrm{H}_{2}$ and $\mathrm{He}$, the mean molecular weight $\mu$ is 2.37, with the elemental abundances of Anders \& Grevesse (1989). The mass of the central star $M_{*}$ is assumed to be $1 M_{\odot}$.

\subsection{Reaction network}

The basic equations for molecular evolution are given by

$$
\frac{\mathrm{d} n(i)}{\mathrm{d} t}=\sum_{j} \alpha_{i j} n(j)+\sum_{j, k} \beta_{i j k} n(j) n(k),
$$

where $n(i)$ is the number density of species $i$, and the $\alpha_{i j}$ and $\beta_{i j k}$ are rate coefficients. We use the "new standard model" network of chemical reactions for the gas-phase chemistry (Terzieva \& Herbst 1998; Osamura et al. 1999). The ionization rate by cosmic rays is assumed to be the "standard" value for molecular clouds, $\zeta=1.310^{-17} \mathrm{~s}^{-1}$ (e.g. Millar et al. 1997), because the attenuation length of cosmic ray ionization is much larger than the column density for $R \geq 50 \mathrm{AU}$. The ionization rate is uncertain by a factor of a few at least (e.g. van der Tak \& van Dishoeck 2000). Calculations with $\zeta=2.610^{-17} \mathrm{~s}^{-1}$ yield molecular column densities that differ by less than a factor of 3 from those with our standard $\zeta$.

We have extended the network to include monodeuterated analogues of hydrogen-bearing species (Millar et al. 1989; Aikawa \& Herbst 1999b). For normal exothermic reactions and dissociative recombination reactions, we have assumed that the total rate coefficient is unchanged for deuterated analogues, and have also assumed statistical branching ratios. There are some exceptions to the statistical rules; for example, the dissociative recombination of $\mathrm{HCND}^{+}$does not produce DCN but HCN. Similar rules are set for the hydrogenation of $\mathrm{HCN}$ and $\mathrm{DCN}$, i.e., $\mathrm{HCN}+\mathrm{H}_{2} \mathrm{DO}^{+}$produces $\mathrm{HCND}^{+}$or $\mathrm{HCNH}^{+}$, but not $\mathrm{DCNH}^{+}$. Another important exception is

$$
\begin{aligned}
\mathrm{H}_{2} \mathrm{CN}+\mathrm{D} & \rightarrow \mathrm{HCN}+\mathrm{HD} \quad(a) \\
& \rightarrow \mathrm{DCN}+\mathrm{H}_{2} \quad(b) .
\end{aligned}
$$

The lower branch $(b)$ is one of the main formation paths for DCN. If we apply the statistical rule, the branching ratio $a / b$ is 2 . However, the experiment by Nesbitt et al. (1990) shows the ratio $a / b=5 \pm 3$. In this paper we assume the ratio $a / b$ to be 5 .

We have included those deuterium exchange reactions for molecular ions and HD that are known to proceed in the laboratory or have been studied in detail theoretically (Millar et al. 1989); such reactions drive the fractionation yet are known to occur for only a few ions since activation energies are common (Henchman et al. 1988).

Although our model does not contain surface chemistry, as does the latest model of Willacy \& Langer (2000), we do include the surface formation of $\mathrm{H}_{2}$ molecules, the surface recombination of ions and electrons, and formation and desorption of ice mantles. As in Paper I, we adopt an artificially low sticking probability $S=0.03$ for adsorbing species on the grain surface, in order to mimic the effect of non-thermal desorption. The sticking coefficient was originally chosen to fit the observed spectrum of $\mathrm{CO}$ emission lines in GG Tau. For thermal desorption from ice mantles, we adopt the same rate coefficients as in Aikawa et al. (1997). The total numbers of species and reactions included in our network are 773 and 10539 , respectively.

The elemental abundances used here are the so-called "low-metal" values (e.g. Lee et al. 1998; Aikawa et al. 1999). The initial molecular abundances are determined by following molecular evolution in a precursor molecular cloud core with physical conditions $n_{\mathrm{H}}=210^{4} \mathrm{~cm}^{-3}$ and $T=10 \mathrm{~K}$ up to $310^{5} \mathrm{yr}$, at which time observed abundances in molecular clouds are reasonably reproduced (Terzieva \& Herbst 1998).

\subsection{X-rays}

Results from the X-ray observation satellites, ROSAT and ASCA, show that $\mathrm{T}$ Tauri stars are strong X-ray emitters, with X-ray luminosities in the range $10^{29}-10^{31} \mathrm{erg} \mathrm{s}^{-1}$ (Montmerle et al. 1993; Glassgold et al. 1997). We include chemical processes caused by X-rays following Maloney et al. (1996) and Glassgold et al. (1997). In Paper I, we showed that $\mathrm{X}$-rays affect the disk chemistry via ionization and induced UV radiation. Direct and secondary ionization of heavy elements by X-rays, which were not taken into account in Paper I, are included in this paper. We find that inclusion of these new processes does not much alter the results of Paper I. For completeness, however, we discuss their inclusion. We adopt an X-ray luminosity of $10^{31} \mathrm{erg} \mathrm{s}^{-1}$, which is almost the upper limit of the observed luminosity, in order to examine the upper limit of the X-ray effects.

\subsubsection{Secondary ionization}

$\mathrm{X}$-rays produce electrons with energies of several hundred $\mathrm{eV}$ by ionizing atoms and molecules. These electrons cause secondary ionization of about 30 molecules and atoms per $\mathrm{keV}$ of primary electron energy, most of which are hydrogen molecules. Secondary ionization is more effective than direct ionization by X-rays in a hydrogen-dominated gas, so that the overall ionization rate per unit time is given approximately by

$\zeta_{\mathrm{x}}=N_{\mathrm{sec}} \int \sigma(E) F(E) \mathrm{d} E$ 
where $N_{\text {sec }}$ is the number of secondary ionizations per unit primary photoelectron energy, $\sigma$ is the cross section for direct X-ray ionization for all elements weighted by their solar abundances, and the X-ray photon flux $F(E)$, in units of per unit area per unit time, is given by

$F(E)=F_{\mathrm{o}}(E) \mathrm{e}^{-\tau(E)}$

where $\tau$ is the optical depth along a path from the central star. The actual value of $\zeta_{\mathrm{x}}$ at a radius of $R=700 \mathrm{AU}$ in the Kyoto model is shown as a function of height from the midplane in Fig. 2 of Paper I. The ionization rate via Xrays is higher than that via cosmic rays in regions roughly above the scale height of the disk.

Although the photoelectrons ionize mostly $\mathrm{H}_{2}$ and $\mathrm{H}$, the secondary ionization of heavy elements may be important for their chemistry. Secondary ionization rate coefficients for heavy elements are estimated by

$\zeta_{\mathrm{x}}^{\mathrm{m}}=\zeta_{\mathrm{x}} \frac{\sigma_{\mathrm{ei}, \mathrm{m}}(E)}{\sigma_{\mathrm{ei}, \mathrm{H}}(E)}$

where $\sigma_{\mathrm{ei}, \mathrm{m}}(E)$ is the electron-impact ionization cross section of element $m$ at energy $E$. We used the cross sections given by Lennon et al. (1988), and averaged the ratio $\sigma_{\mathrm{ei}, \mathrm{m}}(E) / \sigma_{\mathrm{ei}, \mathrm{H}}(E)$ over the energy range $0.1-1 \mathrm{keV}$, because the energy of the primary electron is a few hundred eV. Typically, the ratio is $\sim 3-7$ for elements such as $\mathrm{C}$ (4.29) and $\mathrm{Mg}$ (7.09).

\subsubsection{Direct ionization}

The direct X-ray ionization rates for C, N, O, Si, S, Fe, Na, $\mathrm{Mg}, \mathrm{Cl}$ and $\mathrm{P}$ are calculated at each point of the disk using the X-ray flux obtained from Eq. (6) and the ionization cross sections given by Verner et al. (1993). We assume primary ionization of heavy elements in a molecule leads to a doubly ionized species because of the Auger effect; the doubly ionized species then dissociate into two singly charged ions. We consider this destructive reaction only for simple diatomic molecules, because we do not know the products when polyatomic molecules are dissociated by X-rays. This simplification does not affect our results, because X-ray induced photolysis is much more efficient than direct ionization in terms of molecular destruction.

\subsubsection{UV photolysis induced by $\mathrm{X}$-rays}

Energetic photoelectrons produced by X-rays collide with hydrogen atoms and molecules, and generate UV photons, just as cosmic rays do. Although this process was considered in Paper I, it was not discussed. In $\mathrm{H}_{2}$-dominated regions, the photoreaction rate coefficient for a species $m$ is given by

$R_{\mathrm{m}}=2.6 \psi p_{\mathrm{m}} x_{\mathrm{H} 2} \zeta_{\mathrm{x}}(1-\omega)^{-1} \mathrm{~s}^{-1}$

where $\psi=1.4$ is the ratio of number of Lyman-Werner photons produced per $\mathrm{H}_{2}$ ionization (Maloney et al. 1996).
Since hydrogen is mostly in the molecular form in the region we are interested in, we adopt the constant value $x_{\mathrm{H} 2}=0.5$ in this formula. We can ignore the contribution from hydrogen atoms because atomic hydrogen only dominates in the surface regions, which do not contain many molecules. The coefficient $p_{\mathrm{m}}$ is given by Gredel et al. (1989), and $\omega$ is the grain albedo, which is assumed to be 0.5. For molecules which are not listed in Gredel et al. (1989), we estimate the value of $p_{\mathrm{m}}$ from similar molecules. Following Gredel et al. (1987), we adopt $p_{\mathrm{m}}=10$ for CO. The deuterated hydrogen molecule HD is also subject to photodissociation by induced UV. Because HD is dissociated by lines, just like CO, we assume $p_{\mathrm{m}}=10$ for HD.

In fact, Eq. (8) assumes that induced photons are absorbed by molecules locally; in other words, a spatial gradient of $\zeta_{\mathrm{X}}$ is not considered. Since $\zeta_{\mathrm{X}}$ is higher at larger heights in our model, and since induced photons can be emitted in the vertical direction of the disk, the induced photons could penetrate deeper into the disk than we estimate, especially in the outer radius $(R \gtrsim 500 \mathrm{AU})$ in our lower mass disk, in which the total column density is low $\left(A_{\mathrm{v}} \lesssim 1 \mathrm{mag}\right)$. But a quantitative calculation of this effect requires a 2 -D radiation transfer calculation, which is beyond the scope of this paper.

\subsection{UV radiation}

A protoplanetary disk is irradiated by UV radiation from the external interstellar field and from radiation due to the central star. The UV flux from the central star varies temporally, and at $R=100$ AU the unattenuated UV flux can reach a value $10^{4}$ times higher than the interstellar flux (Herbig \& Goodrich 1986; Imhoff \& Appenzeller 1987; Montmerle et al. 1993). As in Paper I, we utilize this maximum value. The radiation fields from the two sources strike the disk from different directions, and thus suffer different degrees of attenuation. We obtain the attenuation of interstellar UV in terms of the visual extinction $A_{\mathrm{v}}$, by calculating the vertical column density from the disk surface to the height we are interested in, using the relation $A_{\mathrm{v}}=N_{\mathrm{H}} /\left[\begin{array}{lll}1.8 & 10^{21} \mathrm{~cm}^{-2} \mathrm{mag}^{-1}\end{array}\right]$. The attenuation of the stellar $\mathrm{UV}, A_{\mathrm{v}}^{\mathrm{star}}$, is obtained by calculating the column density from the central star. Values of $A_{\mathrm{v}}$ and $A_{\mathrm{v}}^{\text {star }}$ are given in Table 1 of Paper I, as a function of height at $R=700 \mathrm{AU}$ for the Kyoto model. These values of extinction are then put into the photo-rate equations of our network.

In Sect. 4, where we report calculated values for molecular distributions in embedded disks, we consider an additional attenuation of 1-2 mag via ambient gas, while the attenuation of the stellar radiation is not modified.

Although the UV radiation is mainly attenuated by dust, self- and mutual-shielding must be considered for $\mathrm{H}_{2}$ and CO (van Dishoeck \& Black 1988; Lee et al. 1996), so that we must solve for the molecular abundances and the UV attenuation self-consistently. As in Paper I, we solve a one-dimensional slab model at each radius of the 

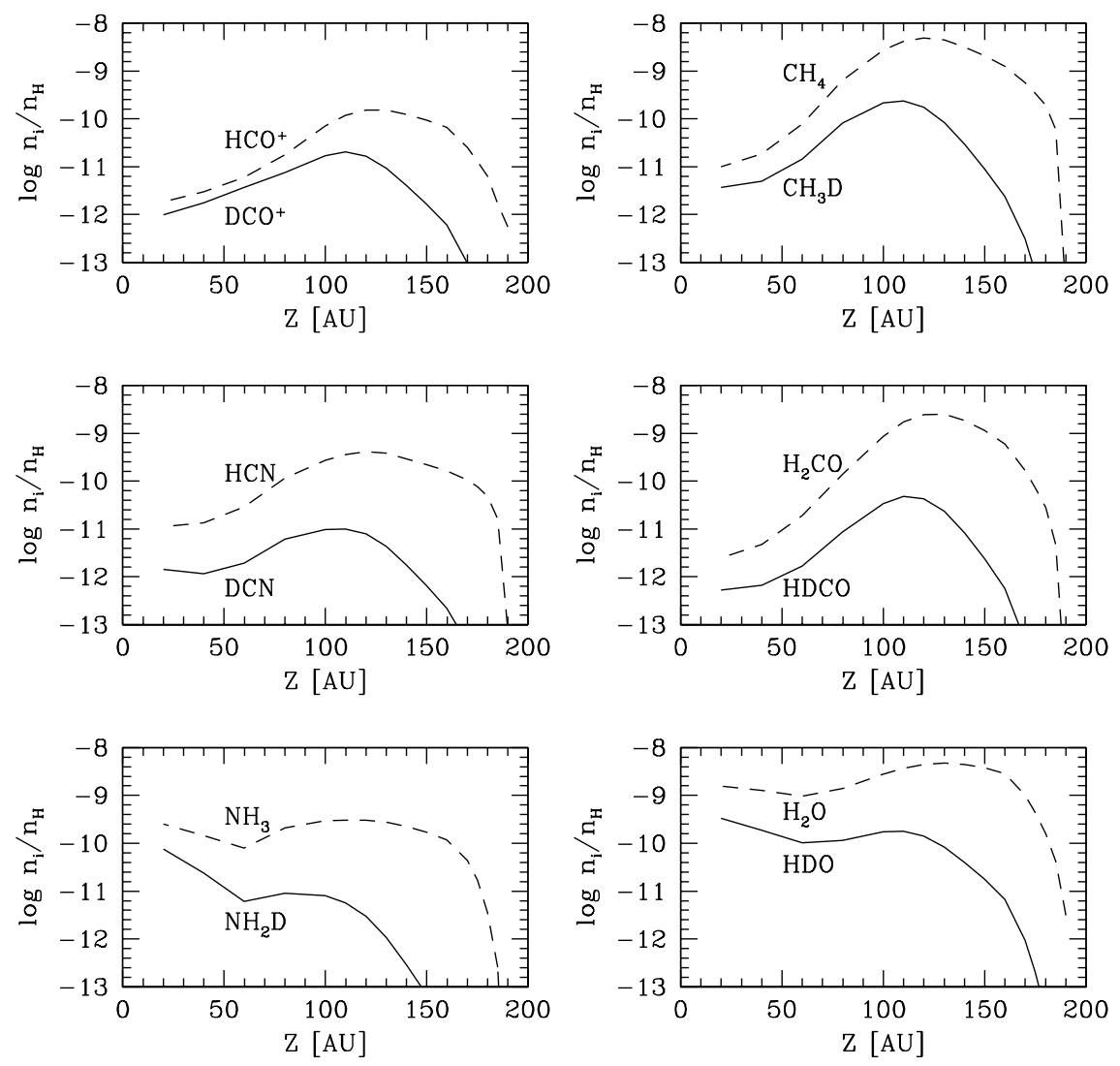

Fig. 1. Vertical distribution of normal (dashed lines) and deuterated (solid lines) molecules at $R=500$ AU. The disk age is assumed to be $t=9.510^{5} \mathrm{yr}$ and the disk mass is less massive than the Kyoto model by an order of magnitude

disk and utilize modified shielding factors from Lee et al. (1996).

\section{Results}

\subsection{Vertical distribution}

Figure 1 shows the vertical distribution of various molecules (dashed lines) and their singly deuterated counterparts (solid lines) at $R=500 \mathrm{AU}$ and at $t=9.510^{5} \mathrm{yr}$, which is a typical age of a $\mathrm{T}$ Tauri star. The disk is assumed to be less massive than the Kyoto model by an order of magnitude. The effects of X-rays from the central star are included. Throughout this paper, we will also present the results of models in which the mass is that of the Kyoto model, in which X-rays are turned off, and in which the time is changed. The low mass model is emphasized because in Paper I, we showed that it is consistent with the observational result of DM Tau by Dutrey et al. (1997).

As can be seen in Fig. 1, most molecules have a peak abundance at some intermediate height; in the surface region of the disk the molecules are dissociated by UV photons both from the interstellar field and the central star, while close to the midplane most molecules are adsorbed onto grains. In the midplane region, however, some gasphase species show a "late-time peak" at $t \sim 10^{5}-10^{6} \mathrm{yr}$ (Ruffle et al. 1997; Paper I) which can occur in gas-grain models with low sticking efficiencies or with non-thermal desorption. The high abundance of $\mathrm{NH}_{3}$ in the midplane is caused by this peak.

A comparison of the concentrations of molecules and their deuterated isotopomers in Fig. 1 shows that molecular $\mathrm{D} / \mathrm{H}$ ratios are much higher than the elemental abundance ratio $\mathrm{D} / \mathrm{H}$ of $1.510^{-5}$. This is not surprising because deuterium fractionation proceeds in a similar way as in molecular clouds. Owing to the energy differences between deuterated species and normal species, and to some rapid exchange reactions, species such as $\mathrm{H}_{3}^{+}$and $\mathrm{CH}_{3}^{+}$have a high $\mathrm{D} / \mathrm{H}$ ratio, and the high ratio propagates to other species through ion-molecule reactions (Millar et al. 1989; Aikawa \& Herbst 1999b). Another route to fractionation lies through the dissociative recombination of molecular ions with high $\mathrm{D} / \mathrm{H}$ ratios, which leads to a high atomic $\mathrm{D} / \mathrm{H}$ ratio. Neutral-neutral reactions involving $\mathrm{H}$ and $\mathrm{D}$ then propagate these high atomic D/H ratios. For example, a major production route for DCN is the reaction between $\mathrm{D}$ and $\mathrm{H}_{2} \mathrm{CN}$.

Figure 1 also shows that molecular $\mathrm{D} / \mathrm{H}$ ratios are higher at smaller heights, where molecules are more heavily depleted from the gas phase onto grain surfaces. Molecular depletion enhances the $\mathrm{D} / \mathrm{H}$ ratio of the remaining gaseous species. For example, $\mathrm{H}_{2} \mathrm{D}^{+}$is formed by the reaction $\mathrm{H}_{3}^{+}+\mathrm{HD}$ and, in many situations, is destroyed mainly by the reaction $\mathrm{H}_{2} \mathrm{D}^{+}+\mathrm{CO}$. Hence the ratio $\mathrm{H}_{2} \mathrm{D}^{+} / \mathrm{H}_{3}^{+}$is proportional to $n(\mathrm{HD}) / n(\mathrm{CO})$, which 

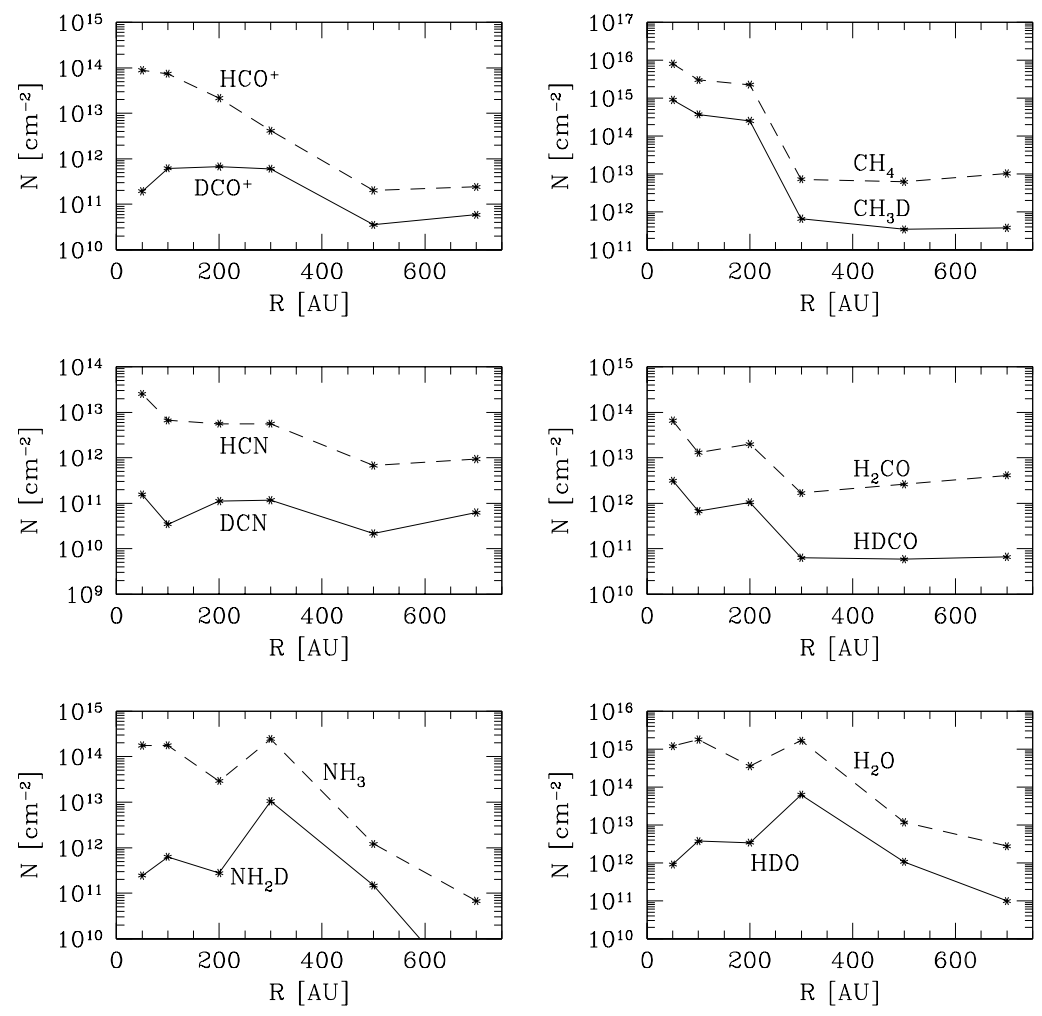

Fig. 2. The column densities of deuterated and normal species as a function of radius. The disk mass is assumed to be less than the Kyoto model by an order of magnitude. The disk age is $t=9.510^{5} \mathrm{yr}$

increases as the gaseous CO abundance decreases (Brown \& Millar 1989). At sufficiently small CO densities, the dominant destruction route for $\mathrm{H}_{2} \mathrm{D}^{+}$becomes reaction with electrons or back reaction with $\mathrm{H}_{2}$.

\subsection{Radial distribution of $D / H$ ratios}

By integrating the vertical distributions of molecular abundances, we obtain molecular column densities. The column densities of deuterated and normal species are shown in Fig. 2 for assorted molecules as a function of disk radius. The time is the same as in Fig. $1: t=9.510^{5} \mathrm{yr}$. Despite the addition of direct and secondary ionization of heavy elements via X-rays in this paper, the calculated column densities of normal species are almost the same as in Paper I. One notable modification concerns the $\mathrm{NH}_{3}$ column density in the outermost region $(R \gtrsim 500 \mathrm{AU})$ of the disk. In Paper I, we assumed that the $\mathrm{H}_{3}^{+}+\mathrm{N}$ reaction produces $\mathrm{NH}^{+}$; however, subsequent information shows that the reaction does not proceed efficiently (Scott et al. 1998). In the outer region, where $\mathrm{N}$ atoms are relatively abundant because of the low density, this reaction was a key component in the synthesis of $\mathrm{NH}_{3}$. At $R=700 \mathrm{AU}$ in the lower mass model with X-rays and $t=9.510^{5} \mathrm{yr}$, the column density of $\mathrm{NH}_{3}$ was $1.210^{12} \mathrm{~cm}^{-2}$ in Paper I, but it is only $6.710^{10} \mathrm{~cm}^{-2}$ in the current model. At smaller radii ( $R \lesssim 300 \mathrm{AU}$ ) the modification is less significant.
We obtained averaged $\mathrm{D} / \mathrm{H}$ ratios for molecules at each radius by dividing the column density of the deuterated species by that of the normal species. Figure 3 shows $\mathrm{D} / \mathrm{H}$ ratios for the lower mass disk model, and Fig. 4 for the Kyoto model. The thick lines show the ratio at $t=9.510^{5} \mathrm{yr}$, and the thin lines at a shorter time of $310^{5} \mathrm{yr}$. The solid lines are for models with X-rays and the dashed lines for models without X-rays.

The radial dependence of the column density ratios shown in Figs. 3 and 4 can be understood from the major mechanism of deuteration. For example, the $\mathrm{D} / \mathrm{H}$ ratios of $\mathrm{NH}_{3}, \mathrm{H}_{2} \mathrm{O}$, and $\mathrm{HCO}^{+}$decrease at $R \lesssim 300 \mathrm{AU}$. These three species are deuterated through $\mathrm{H}_{2} \mathrm{D}^{+}$. Since the exothermicity for the reaction $\mathrm{H}_{3}^{+}+\mathrm{HD} \rightarrow \mathrm{H}_{2} \mathrm{D}^{+}+$ $\mathrm{H}_{2}$ is relatively low $(230 \mathrm{~K})$, the back reaction becomes important as the temperature rises near the star and lowers the $\mathrm{D} / \mathrm{H}$ ratio in the inner regions. On the other hand, the $\mathrm{D} / \mathrm{H}$ ratios in $\mathrm{CH}_{4}$ and $\mathrm{H}_{2} \mathrm{CO}$ do not decrease inwards, because those species are deuterated through $\mathrm{CH}_{2} \mathrm{D}^{+}$, for which the exothermicity of the deuterium exchange reaction is much higher $(370 \mathrm{~K})$ than that for $\mathrm{H}_{2} \mathrm{D}^{+}$. Finally, the DCN/HCN column density ratio slightly decreases inwards because $\mathrm{D}$ atoms are less abundant in inner regions.

$\mathrm{X}$-rays affect the column density ratios. For $R \gtrsim$ $300 \mathrm{AU}$, the $\mathrm{D} / \mathrm{H}$ ratios of $\mathrm{HCO}^{+}, \mathrm{NH}_{3}$ and $\mathrm{H}_{2} \mathrm{O}$ are smaller in the case with $\mathrm{X}$-rays than otherwise, because the enhancement of the $\mathrm{H}_{2} \mathrm{D}^{+}$to $\mathrm{H}_{3}^{+}$ratio is limited by the rate at which $\mathrm{H}_{2} \mathrm{D}^{+}$is destroyed by recombination 

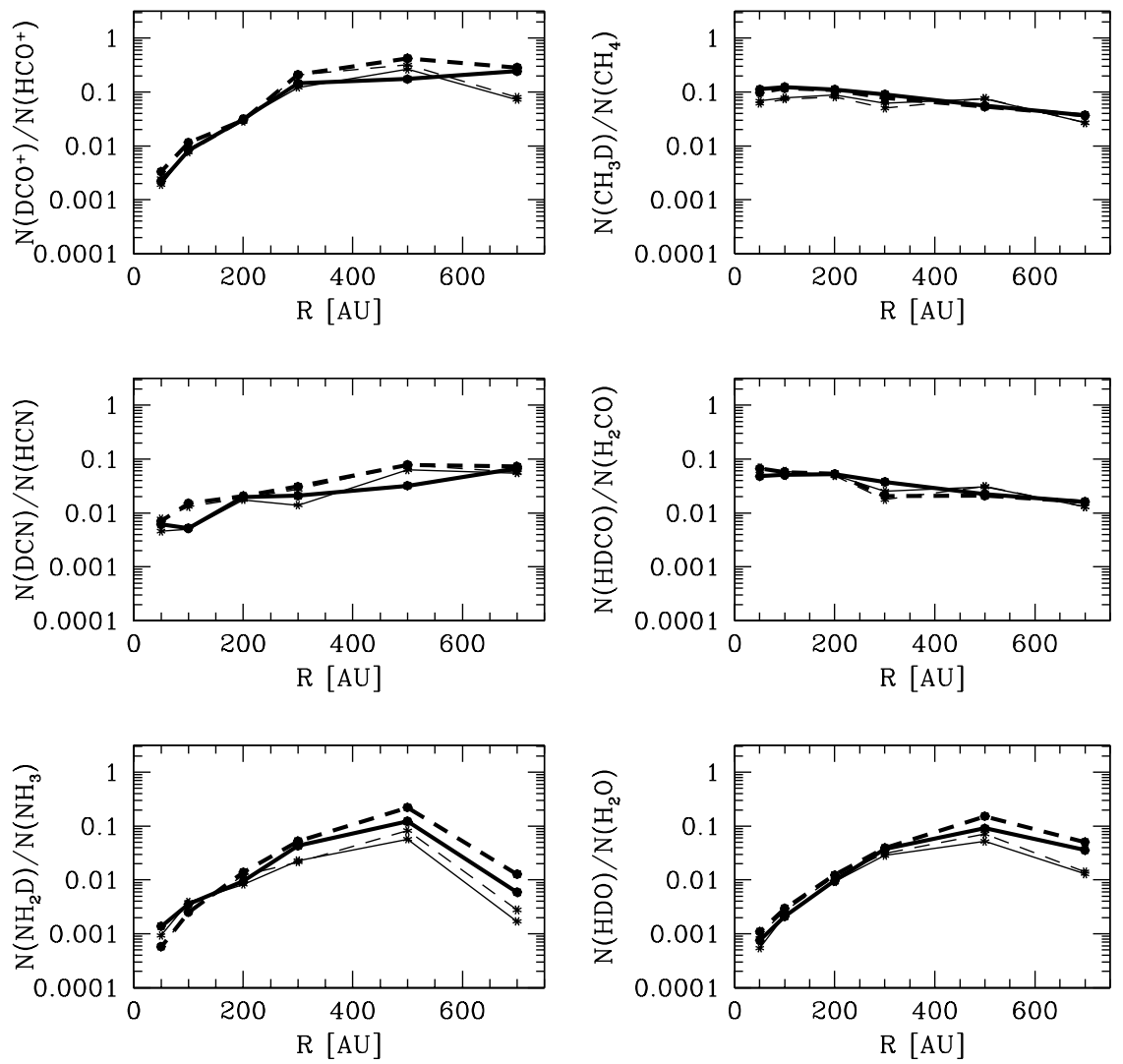

Fig. 3. The column density ratios of deuterated to normal species as a function of radius. The disk mass is assumed to be less than the Kyoto model by an order of magnitude. The thick lines show the ratios at $t=9.510^{5} \mathrm{yr}$, the thin lines at $t=310^{5} \mathrm{yr}$. The solid lines show models with X-rays and the dashed lines without X-rays

with electrons, which are more abundant in the case with X-rays (Guélin et al. 1982; Caselli et al. 1998). In the inner regions, on the other hand, $\mathrm{H}_{2} \mathrm{D}^{+}$is always destroyed more efficiently by the reaction with $\mathrm{CO}$ or $\mathrm{H}_{2}$, and thus the ratios are less dependent on the electron abundance.

The $\mathrm{D} / \mathrm{H}$ ratios also depend on the total mass of the disk. Comparing Fig. 3 with Fig. 4, we can see that, except for $\mathrm{DCO}^{+} / \mathrm{HCO}^{+}$, the ratios are higher in the Kyoto model, especially in the outer region. This mass dependence is caused by molecular depletion onto grains, which is more efficient in disks with higher mass. This dependence does not appear for $\mathrm{HCO}^{+}$, because its abundance peaks at a larger height than those of neutral species, at which $\mathrm{D} / \mathrm{H}$ ratios are less affected by depletion.

So far, detection of deuterated species has been reported only in the disk around LkCa15, where Qi (2000) observed DCN and HDO using the OVRO interferometer (see also Qi et al. 1999). He found DCN and HCN to be distributed within a radius of $\sim 1^{\prime \prime}$ (140 AU at the distance of $140 \mathrm{pc}$ ) and $\sim 3^{\prime \prime}-4^{\prime \prime}$ from the central star, respectively. The differing sizes of the distributions reflect the fact that the only DCN line detected is the $J=3-2$ transition. The column density of DCN was estimated to be $110^{13} \mathrm{~cm}^{-2}$ from the integrated intensity of $\operatorname{DCN}(J=3-2)$, while the column density of $\mathrm{HCN}$ was estimated to be $\sim 10^{15} \mathrm{~cm}^{-2}$ from the intensities of the $\mathrm{H}^{13} \mathrm{CN} J=3-2$ and $J=1-0$ lines. Our model result for the $\mathrm{DCN} / \mathrm{HCN}$ ratio within
$R \lesssim 200 \mathrm{AU}$ is consistent with the observation, independent of the disk mass. However, the absolute column densities of HCN and DCN in the model are significantly smaller than observed. In the region of radius $\lesssim 200 \mathrm{AU}$, the column density of DCN is $\sim 10^{11} \mathrm{~cm}^{-2}$ in our low mass disk model (Fig. 2) and is $\lesssim 10^{12} \mathrm{~cm}^{-2}$ in the Kyoto model (see Fig. 4 and Paper I). Moreover, the column densities of various molecules detected in LkCa15 are significantly higher than those in DM Tau (Qi 2000). One possible explanation for this difference would be the disk mass. The mass of the disk around LkCa15 is estimated to be $0.2 M_{\odot}$ from the dust continuum, which traces the region of radius $\sim 100$ AU, while the mass of Kyoto model within $100 \mathrm{AU}$ is $0.024 M_{\odot}$. In addition to modifying the Kyoto model to include higher masses, the major possibilities for improving our absolute column densities are (a) to lower the artificial sticking probability used in our current model $(S=0.03)$ so that more material remains in the gas phase at a given time, or (b) to include specific non-thermal desorption mechanisms (Willacy \& Langer 2000), and to consider the variability of their efficiency among disks. Finally, a K5 star HD284589, located close to LkCa15, may affect the molecular abundances in the disk through heating and/or UV radiation. These are prospects for future work. Observations of deuterated species in DM Tau would be desirable to compare with our current calculated values. 

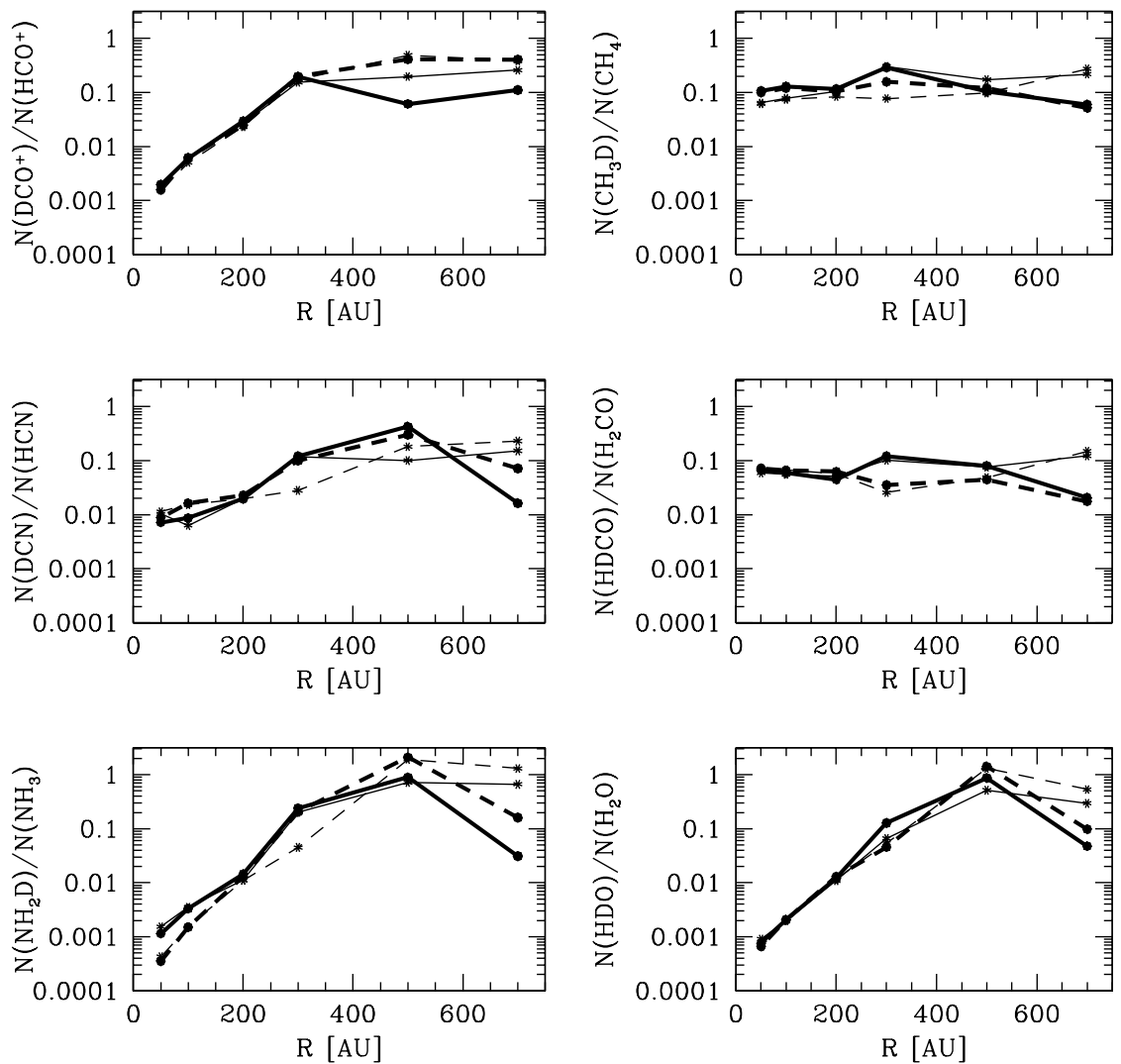

Fig. 4. The column density ratios of deuterated to normal species as a function of radius, but for the Kyoto model. The thick lines show the ratios at $t=9.510^{5} \mathrm{yr}$, the thin lines at $t=310^{5} \mathrm{yr}$. The solid lines show models with X-rays and the dashed lines without X-rays

Like DCN, the estimated column density of HDO in LkCa15 - (2-7) $10^{14} \mathrm{~cm}^{-2}$ (Qi 2000) - is higher than the value $\left(\lesssim 110^{14} \mathrm{~cm}^{-2}\right)$ obtained in our models. Since $\mathrm{H}_{2} \mathrm{O}$ cannot be observed from the ground, the $\mathrm{HDO} / \mathrm{H}_{2} \mathrm{O}$ ratio is not determined. It is interesting that the intensity peak of HDO is offset from the central star, which is consistent with our model.

\section{Disks embedded in ambient gas}

Statistical observations of molecular emission lines in disks are important in order to determine their size, time scale of gas dissipation, and chemical abundances. Several gaseous disks have been found via surveys of $\mathrm{CO} J=2-1$ and $J=1-0$ lines (Kawabe et al. 1993; Koerner et al. 1993; Dutrey et al. 1994; Koerner \& Sargent 1995). The merit of using CO lines is that the molecule is in general much more abundant than any other molecules with heavy elements in interstellar conditions. However, the number of disks observed via molecular lines is much smaller than that via dust continuum studies. One of the difficulties in searching for gaseous disks via $\mathrm{CO}$ lines is contamination of the lines with ambient cloud gas. CO is abundant not only in the disk, but also in ambient clouds, and the critical densities of the excitation of its $J=2-1$ and $J=1-0$ lines are comparable to the cloud gas density. Hence it is useful if we can find molecular lines which preferentially trace the gas in disks. Such lines have to satisfy the following two con- ditions: (a) their critical densities should be higher than a typical density of molecular clouds $\left(n_{\mathrm{H}} \sim 10^{4} \mathrm{~cm}^{-3}\right)$ and (b) the molecule should be reasonably abundant within the disk. In fact, there are many molecular rotational lines, especially the high $-J$ lines, the critical density of which is higher than the cloud density but lower than or comparable to the disk density. The latter condition (b) can be checked based on our model.

In our calculation of molecular column densities in disks in Paper I, however, we assumed the disks to be directly exposed to the interstellar radiation field. In order to see if there is any molecular line which can be used to trace embedded disks, we have to reconsider the problem including the UV attenuation via ambient gas. One possible tracer is $\mathrm{CN}$, the rotational $(N=2-1)$ transition of which is one of the strongest lines detected in the disks around DM Tau, GG Tau, and LkCa15 (Dutrey et al. 1997; Qi 2000). The clear detection of the line indicates that $\mathrm{CN}$ is relatively abundant in these disks, and the high critical densities $\left(n_{\mathrm{H}}=310^{5} \mathrm{~cm}^{-3}\right.$ for $N=1-0$ and $10^{6} \mathrm{~cm}^{-3}$ for $N=2-1$ ) of the lines seem ideal in order to avoid contamination with ambient clouds at lower density. But theoretical studies show that CN exists mostly in the surface regions of disks, in which interstellar UV plays a dominant role in the chemistry (Paper I), and thus it is not clear if it is also abundant in disks shielded from interstellar radiation. In this section, we discuss the column 

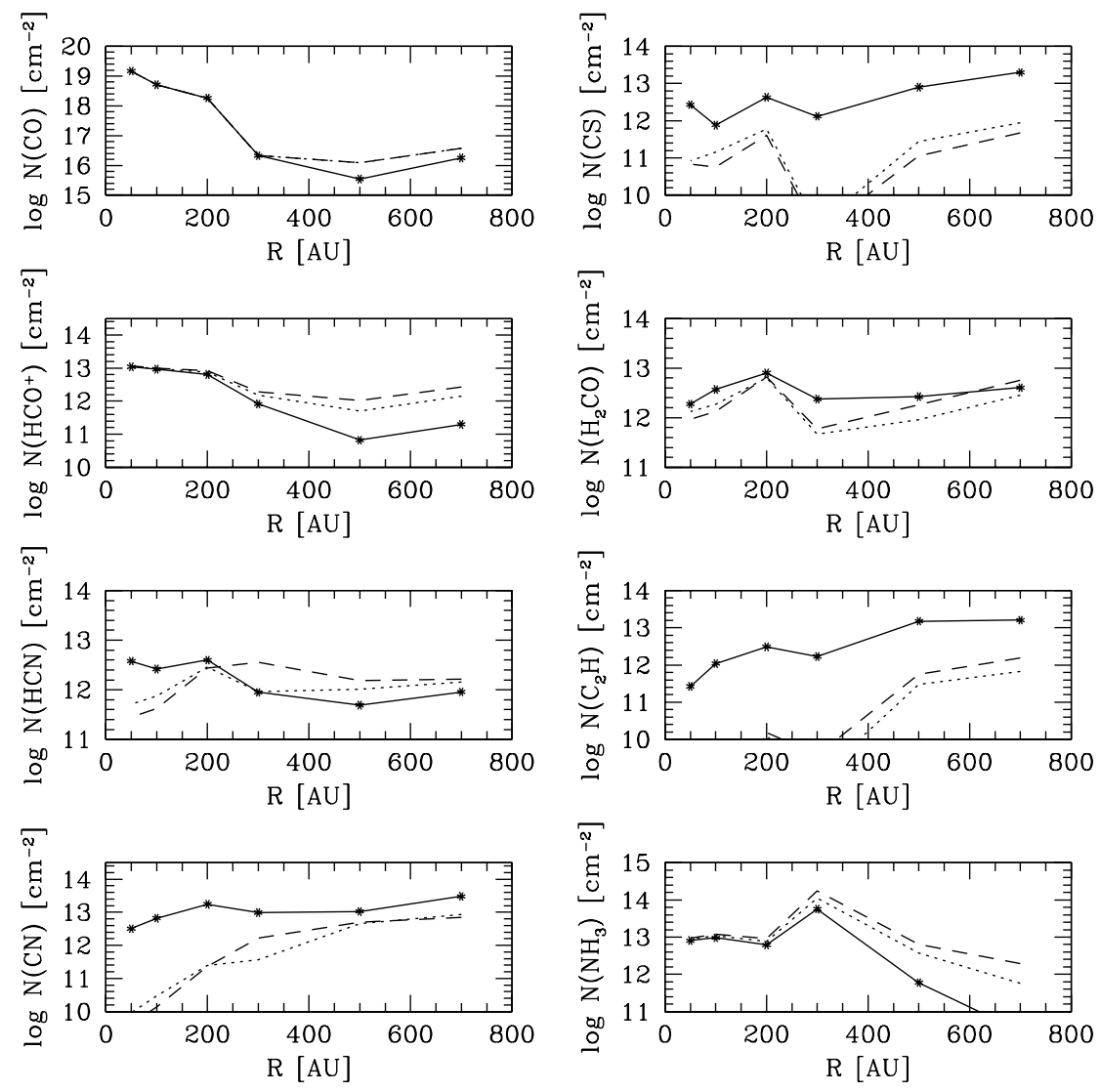

Fig. 5. Column densities of assorted molecules as a function of radius. The disk mass is assumed to be less than the Kyoto model by an order of magnitude, and the disk age is $t=9.510^{5}$ yr. Ionization, dissociation, and induced photolysis by X-rays are not considered. The solid lines show results for disks directly exposed to the interstellar UV field, while the dashed and dotted lines show results for disks embedded in ambient gas of $A_{\mathrm{v}}=1$ and 2, respectively

densities of $\mathrm{CN}$, together with other species, in embedded disks.

Figures 5 and 6 show calculated radial column density distributions of assorted species in the lower mass disk model at $t=9.510^{5} \mathrm{yr}$. Figure 5 contains model results in the absence of any X-ray emission from the central star, while Fig. 6 shows results when X-ray processes are included. The solid lines, dotted lines, and dashed lines are for $A_{\mathrm{v}}=0,1$, and $2 \mathrm{mag}$, respectively. It is easy to see that the distributions can depend on both the X-ray irradiation and the degree of extinction. In the model without $\mathrm{X}$-rays, the column densities of radicals such as $\mathrm{CN}$ and $\mathrm{C}_{2} \mathrm{H}$ are extremely sensitive to the existence of ambient gas above and below the disk since this gas interferes with the photodissociation that produces the radicals. The effect is especially drastic at inner $(R \lesssim 300$ AU) radii, where the gas density is higher and thus abundances of radical species are very low without photoprocesses. It should be noted, however, that the column density of $\mathrm{CN}$ in the case with $A_{\mathrm{v}}=2 \mathrm{mag}$ is still reasonably large at $R \gtrsim 300$ AU. We additionally calculated molecular abundances at $R=500 \mathrm{AU}$ for $A_{\mathrm{v}}=4 \mathrm{mag}$, in which the $\mathrm{CN}$ column density is $3.710^{12} \mathrm{~cm}^{-2}$. These results suggest that $\mathrm{CN}$ can be a disk tracer at least in the outer regions $(R \gtrsim 300 \mathrm{AU})$ for $\mathrm{T}$ Tauri stars without high X-ray luminosity.
In the model with $\mathrm{X}$-rays from the central star, the column densities of radicals such as $\mathrm{CN}, \mathrm{CH}$, and $\mathrm{C}_{2} \mathrm{H}$ are high even in the inner regions. The high ionization rate and induced photolysis by X-rays significantly enhance the radical species in regions with small radius and large height. We should note that the column densities of those radicals obtained in this model are upper limits because the X-ray luminosity we assumed $-L_{\mathrm{x}}=10^{31} \mathrm{erg} \mathrm{s}^{-1}$ - is close to the upper limit of the temporally varying $\mathrm{X}$-ray intensity from $\mathrm{T}$ Tauri stars. But sufficient column densities of the radicals are expected in the inner regions $(\lessgtr 200 \mathrm{AU})$ even if the average X-ray luminosity is lower by an order of magnitude, since their radical column densities roughly depend linearly on the X-ray luminosity there. Therefore $\mathrm{CN}$ can trace even the inner regions $(R \lesssim 200 \mathrm{AU})$ of embedded disks when there is some X-ray luminosity.

As for other species, it is interesting to note that the column densities of $\mathrm{HCO}^{+}$and $\mathrm{NH}_{3}$ tend to be higher in disks with non-zero $A_{\mathrm{v}}$, regardless of the X-ray luminosity, which suggests that their high frequency transitions with high critical densities can also be tracers of embedded disks. The centrally peaked distribution of $\mathrm{HCO}^{+}$makes it the better tracer because the line can be more sensitive to a disk with small radius. 

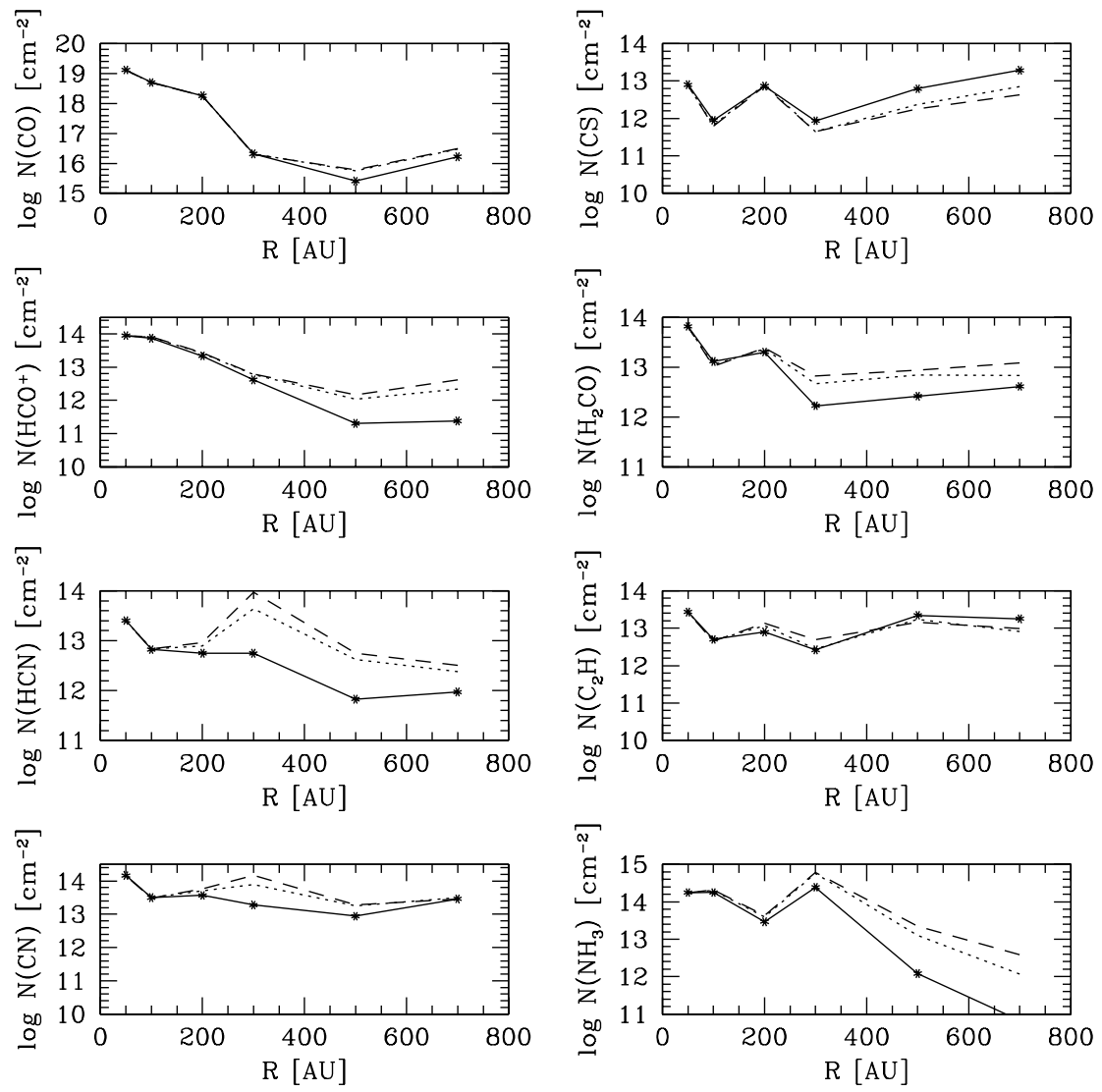

Fig. 6. Same as in Fig. 5, but with X-rays from the central star included

Although the calculations reported in this section deal with the effect of embedding disks in clouds, a close observation of Figs. 5 and 6 shows that some qualitative trends are actually independent of the degree of extinction. For example, species such as $\mathrm{CN}, \mathrm{C}_{2} \mathrm{H}$, and $\mathrm{H}_{2} \mathrm{CO}$ show a central peak in the case with $\mathrm{X}$-ray irradiation and a central hole in the case of no such irradiation regardless of whether or not the disk is embedded. This behavior is not universal; the HCN distribution shows a similar dependence on the X-ray luminosity only when the disks are embedded. Specifically, in the model without X-ray irradiation, HCN (in the case of $A_{\mathrm{v}} \geq 1 \mathrm{mag}$ ) shows a central hole of radius $\sim 200 \mathrm{AU}$, while in all cases with X-ray irradiation it is centrally peaked. Thus, if X-ray fluxes are different from one disk to another, one can expect the radial distributions of individual molecules to be different. Interestingly, interferometric observations show that $\mathrm{CN}$ is centrally peaked in the disk around DM Tau (Dutrey 2000, personal communication) but exhibits a central hole in the disk around LkCa15 (Qi 2000).

\section{Summary}

We have presented two major extensions from our previous models reported in Paper I.

Firstly, we have investigated the two-dimensional distribution of deuterated species in protoplanetary disks. The molecular $\mathrm{D} / \mathrm{H}$ ratios in gaseous disks evolve in a similar way as in molecular clouds. In the vertical direction the $\mathrm{D} / \mathrm{H}$ ratios are higher at lower height, where molecules are more heavily depleted onto grain surfaces.

The $\mathrm{D} / \mathrm{H}$ ratios of $\mathrm{HCO}^{+}, \mathrm{NH}_{3}$, and $\mathrm{H}_{2} \mathrm{O}$ decrease significantly towards smaller radii at $R \lesssim 300 \mathrm{AU}$. These molecules are deuterated through $\mathrm{H}_{2} \mathrm{D}^{+}$, the abundance of which is rather sensitive to the temperature, which increases at small radii. The $\mathrm{D} / \mathrm{H}$ ratios of $\mathrm{CH}_{4}$ and $\mathrm{H}_{2} \mathrm{CO}$ do not decrease inwards because these species are deuterated through $\mathrm{CH}_{2} \mathrm{D}^{+}$, which is formed from $\mathrm{CH}_{3}^{+}$ through a sufficiently exothermic reaction that it does not possess much of a temperature dependence in the range $(T \lesssim 40 \mathrm{~K})$ we are concerned with in this paper. The $\mathrm{DCN} / \mathrm{HCN}$ ratio is higher at larger radii because DCN is formed primarily from $\mathrm{H}_{2} \mathrm{CN}$ via the reaction with $\mathrm{D}$ atoms, which are more abundant at larger radii, where the density is lower.

The $\mathrm{D} / \mathrm{H}$ ratios depend not only on the temperature, but also on the disk mass and the X-ray flux. In the disk with larger mass, the column-density ratios of deuterated species to normal species are generally higher because of heavier depletion of molecules onto grains. With a higher X-ray flux, the $\mathrm{D} / \mathrm{H}$ ratios of $\mathrm{HCO}^{+}, \mathrm{NH}_{3}$ and $\mathrm{H}_{2} \mathrm{O}$ are smaller at large radial distances because the enhancement of the $\mathrm{H}_{2} \mathrm{D}^{+}$to $\mathrm{H}_{3}^{+}$ratio is reduced by more copious electrons.

Our results for the ratio of the column densities of DCN and HCN are in reasonable agreement with the observation of the disk around LkCa15 (Qi 2000) whether 
we utilize the Kyoto or the low mass model, but the absolute column densities we calculate are too low for this rather massive disk.

Secondly, we have investigated molecular column densities in disks embedded in ambient clouds using models without and with X-rays from the central T Tauri star.

In the models without X-rays, the column densities of radicals such as $\mathrm{CN}$ are sensitive to the attenuation of interstellar UV by ambient gas, especially in the inner radius of the disk. In the disk with direct UV irradiation, the $\mathrm{CN}$ column density is $310^{12}-310^{13} \mathrm{~cm}^{-2}$ in the region $50 \leq R \leq 700 \mathrm{AU}$, while in the disk embedded in ambient gas, the column density of $\mathrm{CN}$ is $10^{12}-10^{13} \mathrm{~cm}^{-2}$ in the outer region of the disk $(R \gtrsim 300 \mathrm{AU})$, but decreases for radii $\lesssim 300$ AU by orders of magnitude. On the other hand, in the models with X-rays, ionization and induced photolysis by X-rays enhance the abundance of $\mathrm{CN}$ and other radicals so that the column density of $\mathrm{CN}$ is as high as $\gtrsim 10^{13} \mathrm{~cm}^{-2}$ at $50 \leq R \leq 700 \mathrm{AU}$. Since the X-ray flux is higher in inner regions, radial distributions of radicals such as CN are centrally peaked. Indeed, the distributions for non-radicals tend to show a similar effect.

The molecular ion $\mathrm{HCO}^{+}$is centrally peaked regardless of the X-ray luminosity, and is more abundant in the case with UV shielding via ambient clouds. Inclusion of X-rays further enhances the column density of $\mathrm{HCO}^{+}$.

Our calculated results, combined with the fact that $\mathrm{CN}$ and $\mathrm{HCO}^{+}$are clearly detected in the non-embedded disks around DM Tau, GG Tau, and LkCa15, suggest that CN and $\mathrm{HCO}^{+}$can be gaseous disk tracers towards embedded objects especially with high X-ray luminosity. Our results also suggest that the radial distribution of the radicals $\mathrm{CN}$ and $\mathrm{C}_{2} \mathrm{H}$ and some non-radical species $\left(\mathrm{HCN}\right.$ and $\mathrm{H}_{2} \mathrm{CO}$ ) may vary among disks depending on the $\mathrm{X}$-ray luminosity of the central star.

Acknowledgements. The authors are grateful to A. Dutrey, G. Blake, C. Qi, and M. Saito for stimulating discussions on the line observation of protoplanetary disks. Y. A. is grateful for financial support from the Japan Society for Promotion of Science. The Astrochemistry Program at The Ohio State University is supported by The National Science Foundation. Numerical calculations were partly carried out at the Astronomical Data Analysis Center of the National Astronomical Observatory of Japan, and on the Cray T90 at the Ohio Supercomputer Center.

\section{References}

Adams, F. C., \& Lin, D. N. C. 1993, in Protostars and Planets III, ed. E. H. Levy, \& J. I. Lunine (Tucson: Univ. of Arizona Press), 721

Aikawa, Y., \& Herbst, E. 1999a, A\&A, 351, 233 (Paper I)

Aikawa, Y., \& Herbst, E. 1999b, ApJ, 526, 314

Aikawa, Y., Umebayashi, T., Nakano, T., \& Miyama, S. M., 1997, ApJ, 486, L51

Aikawa, Y., Umebayashi, T., Nakano, T., \& Miyama, S. M. 1999, ApJ, 519, 705

Anders, E., \& Grevesse, N. 1989, Geochimica et Cosmochimica Acta, 53, 197
Beckwith, S. V. W., Sargent, A. I., Chini, R. S., \& Güsten, R. 1990, AJ, 99, 924

Brown, P. D., \& Millar, T. J. 1989, MNRAS, 237, 661

Cameron, A. G. W. 1973, Space Sci. Rev., 15, 121

Caselli, P., Walmsley, C. M., Terzieva, R., \& Herbst, E. 1998, ApJ, 499, 234

Dutrey, A., Guilloteau, S., \& Guélin, M. 1997, A\&A, 317, L55

Dutrey, A., Guilloteau, S., \& Simon, M. 1994, A\&A, 286, 149

Glassgold, A. E., Najita, J., \& Igea, J. 1997, ApJ, 480, 344

Gredel, R., Lepp, S., \& Dalgarno, A. 1987, ApJ, 323, L137

Gredel, R., Lepp, S., Dalgarno, A., \& Herbst, E. 1989, ApJ, 347,289

Guélin, M., Langer, W. D., \& Wilson, R. W. 1982, A\&A, 107, 10

Guilloteau, S., \& Dutrey, A. 1998, A\&A, 339, 467

Handa, T., Miyama, S. M., Yamashita, T., et al. 1995, ApJ, 449,894

Hayashi, C. 1981, Prog. Theor. Phys. Suppl., 70, 35

Henchman, M. J., Paulson, J. F., Smith, D., Adams, N. G., \& Lindinger, W. 1988, in Rate Coefficients in Astrochemistry, ed. T. J. Millar, \& D. A. Williams (Dordrecht: Kluwer), 201

Herbig, G. H., \& Goodrich, R. W. 1986, ApJ, 309, 294

Imhoff, C. L., \& Appenzeller, I. 1987, in Scientific Accomplishments of the I. U. E., ed. Y. Kondo (Dordrecht: Reidel), 295

Kawabe, R., Ishiguro, M., Omodaka, T., Kitamura, Y., \& Miyama, S. M. 1993, ApJ, 404, L63

Koerner, D. W., \& Sargent, A. I. 1995, AJ, 109, 2138

Koerner, D. W., Sargent, A. I., \& Beckwith, S. V. W. 1993, Icarus, 106, 2

Lee, H.-H., Herbst, E., Pineau des Forêts, G., Roueff, E., \& Le Bourlot, J. 1996, A\&A, 311, 690

Lee, H.-H., Roueff, E., Pineau des Forêts, G., et al. 1998, A\&A, 334, 1047

Lennon, M. A., Bell, K. L., Gilbody, H. B., et al. 1988, J. Phys. Chem. Ref. Data, 17, 1285

Maloney, P., Hollenbach, D. J., \& Tielens, A. G. G. M. 1996, ApJ, 466, 561

Millar, T. J., Bennett, A., \& Herbst, E. 1989, ApJ, 340, 906

Millar, T. J., Farquhar, P. R. A., \& Willacy, K. 1997, A\&AS, 121,139

Montmerle, T., Feigelson, E. D., Bouvier, J., \& André, P. 1993, in Protostars and Planets III, ed. E. H. Levy, \& J. I. Lunine (Tucson: Univ. of Arizona Press), 689

Nesbitt, F. L., Marston, G., \& Stief, L. J. 1990, J. Phys. Chem., 94,4946

Osamura, Y., Fukuzawa, K., Terzieva, R., \& Herbst, E. 1999, ApJ, 519, 697

Qi, C. 2000, Ph.D. Thesis, California Institute of Technology

Qi, C., Blake, G. A., \& Sargent, A. I. 1999, in Science with the Atacama Large Millimeter Array (ALMA), a meeting held October 6-8, 1999 at Carnegie Institution of Washington

Ruffle, D. P., Hartquist, T. W., Taylor, S. D., \& Williams, D. A. 1997, MNRAS, 291, 235

Saito, M., Kawabe, R., Ishiguru, M., et al. 1995, ApJ, 453, 384

Scott, G. B. I., Fairley, D. A., Freeman, C. G., McEwan, M. J., \& Anicich, V. G. 1998, J. Chem. Phys., 109, 9010

Terzieva, R., \& Herbst, E. 1998, ApJ, 501, 207

van der Tak, F. F. S., \& van Dishoeck, E. F. 2000, A\&A, 358, L79

van Dishoeck, E. F., \& Black, J. H. 1988, ApJ, 334, 771

Verner, D. A., Yakovlev, D. G., Band, I. M., \& Trzhaskovskaya, M. B. 1993, At. Data Nucl. Data Tables, 55, 233

Willacy, K., \& Langer, W. D. 2000, ApJ, 544, 903 